\title{
基于时序遥感的庐山自然保护区 植被分类及其变化分析
}

\author{
张 琍 ${ }^{1}$, 李 斌 ${ }^{1,2}$, 阳文静 ${ }^{1}$, 李 琼 $^{3}$
}

(1. 江西师范大学鄱阳湖湿地与流域研究教育部重点实验室, 南昌 330022;

2. 中国石油大学(华东)海洋与空间信息学院, 山东青岛 266580; 3. 武汉市测绘研究院, 武汉 430022)

\begin{abstract}
摘 要: 自然保护区是生物多样性最丰富的区域, 针对近年来庐山自然保护区森林生物多样性进行调查, 了解保护 区内植被的分布及其变化, 对于保护庐山境内的林业资源具有重要的意义。论文利用2015-2018年Proba-V卫星 $100 \mathrm{~m}$ 分辨率 $5 \mathrm{~d}$ 植被指数产品, 使用最大值合成法、Hants 时间序列谐波分析方法对数据进行平滑降噪处理, 并采 用随机森林算法进行分类, 同时对启山林地变化进行分析。结果表明: (1) 2015-2018年庐山林地随机森林分类的 总体精度分别为 $85.00 \% 、 84.25 \% 、 84.75 \% 、 85.25 \%, \mathrm{Kappa}$ 系数分别为 $0.80 、 0.79 、 0.81 、 0.80$ 。分类结果与 LC-CCI、 Globcover、Globeland30、MODIS 等植被覆盖分类产品的对比分析表明, 基于时序 NDVI产品的随机森林分类器在 空间分辨率和制图精度上都取得了较好的分类效果和较高的分类精度。(2) 庐山自然保护区内针叶林、阔叶林分 布较为集中, 混交林与竹林零散分布, 其中, 针叶林的分布面积最大, 占比 $39.36 \%$; 竹林的分布面积最小, 占比为 $14.63 \%$ 。(3) 2015-2018 年间,庐山林地类型分布变化不大, 阔叶林、针叶林、混交林变化率均低于 $10 \%$, 阔叶林变 化最小,竹林由于受到人类活动影响, $4 \mathrm{a}$ 间变化幅度波动较大。
\end{abstract}

关 键 词: Proba-V; 随机森林; 森林植被制图与分类; 时空分布; 庐山

森林被人们誉为“地球之肺”, 是陆地生态系统 的重要组成部分, 起着维持地气系统物质和能量平 衡、陆地生态系统稳定的重要功能,对于人类的生 存和发展有着极其重要的作用 ${ }^{[1]}$ 。尽管自然保护区 处于保护状态, 但由于气候变化和周边人类活动对 保护区的影响 [2],准确地调查和绘制森林用地类型 对于保持保护区内生境和生物多样性的完整性具 有重要的作用。庐山森林保护区是世界文化景观 遗产地和世界地质公园, 同时区域内人类活动频 繁[3]。近年来, 由于气候变化及人类活动的影响, 庐 山自然保护区植被群落类型也受到了一定影响, 对
保护区内的植被群落类型及其分布进行调查并探 讨其时空变化规律,对保护并合理利用保护区内森 林资源、保持保护区内生境和生物多样性的完整性 具有重要的意义。

传统的森林植被调查主要依靠人工实地调查, 周期长、成本高, 且耗费大量的人力和物力。遥感 技术具有覆盖范围广、信息量大和更新周期短等 特点 ${ }^{[4-5]}$, 为植被制图和林业资源的调查监测提供 了有力的技术支撑。利用遥感进行森林植被制图 与监测的技术已经非常成熟, 多光谱数据 ${ }^{[6-8]}$ 、高光 谱数据 ${ }^{[0] 、}$ 、高空间分辨率数据 ${ }^{[10]}$ 、雷达数据都被用于

收稿日期 : 2020-03-04; 修订日期 : 2020-10-16。

基金项目: 国家自然科学基金青年项目(41701514,41761076); 江西省自然科学基金项目(20161BAB213074); 鄱阳湖湿地与流 域研究教育部重点实验室开放基金(PK20170005)。[Foundation: National Natural Science Foundation of China, No. 41701514 and 41761076; Natural Science Foundation of Jiangxi Province, No. 20161BAB213074; Open Fund of Key Laboratory of Poyang Lake Wetland and Watershed Research, Ministry of Education (Jiangxi Normal University), No. PK20170005. ]

第一作者简介: 张琍 $1981-)$, 女, 湖北武汉人, 博士, 副教授, 主要从事雷达与光学遥感应用、植被遥感分类等研究。 E-mail: zhanglinu@126.com $\mathrm{Li}$, Li Bin, Yang Wenjing, et al. Forest vegetation classification and its variation in Lushan Nature Reserve using Proba-V vegetation products. Progress in Geography, 2021, 40(4): 703-712. ] DOI: 10.18306/dlkxjz.2021.04.014 
森林植被分类与制图研究。高光谱数据由于其波 段多, 分类结果较为精确。激光雷达数据与机载高 光谱数据(CASI)的融合, 可以将树种高度和地表信 息相结合, 提高分类精度 ${ }^{[11]}$ 。但高光谱数据和雷达 数据的难获取性和时效特性, 导致此类数据在森林 分类及其变化监测研究中受到限制。多光谱数据 则成为了有效的替代数据, 但由于多光谱数据波段 较少, 植被相关仅 1 2个, 单景数据很难进行植被群 落精细分类。由于植被群落物候变化, 长时序的多 光谱数据成为了较为有效的方法。研究表明, 利用 多时相MODIS 数据提取的物候变化来进行植被群 落分类效果要优于单景的多光谱数据 ${ }^{[12]}$ 。但由于 其空间分辨率较低, 适合于全球范围的土地覆盖研 究, 而对区域植被群落信息提取则作用有限。Proba- $V$ 数据作为专门的植被卫星产品, 其时间与空间 分辨率都非常适合于区域植被群落信息的提取。 Zhang 等 ${ }^{[13]}$ 基于 Proba- $\mathrm{V}$ 时间序列数据对红星农场 的作物进行制图, 研究表明, 时间序列的 Proba- $\mathrm{V}$ 数 据可以作为可靠的植被识别和面积估计的有用 来源。

在分类方法选取中, 监督分类、决策树分类、机 器学习分类及面向对象分类方法常常被用于植被 分类研究中 ${ }^{[14]}$, 其中面向对象分类法适合于高分辨 率的图像分析, 支持向量机、随机森林等分类由于 其高稳定性、可处理高维特征、训练速度快、无偏估 计等特点, 成为了目前植被精细分类研究方法中的 重要方法 ${ }^{[14-15]}$ 。张赫林等 ${ }^{[16]}$ 基于 Landsat 时间序列数 据,采用光谱扩展与基于回归树的决策树分类 (CART) 获取规则的决策树分类方法, 对各类土地利 用类型近 $30 \mathrm{a}$ 的变化情况进行分析。关于庐山植 被的研究, 学者们主要从群落学角度调查分析了庐 山植被的主要类型 ${ }^{[17-18]}$ 、垂直地带性 ${ }^{[19]}$ 及外来物种 ${ }^{[20]}$ 等几个方面。但尚缺乏对庐山植被定期监测的有 效方法与植被的时空动态变化分析。植被群落的 演替变化关系到生态系统的稳定性, 进而影响到生 态系统多样性及生态安全。如何快速、准确地进行 森林植被制图并研究其变化, 是目前吕需解决的关 键问题。

本文研究基于长时序的植被指数数据和样点 数据, 采用随机森林分类方法对庐山自然保护区的 林地进行分类研究并分析其变化, 旨在研究一套简 单、易获取的、高精度的遥感图像森林植被分类方 法, 可以定期为庐山自然保护区提供有效的植被群
落制图产品,为全面掌握庐山自然保护区典型林地 类型提供基础信息; 并在此基础上通过土地利用转 移矩阵, 分析植被群落的时空变化, 为研究庐山自 然保护区植被群落的演替过程和自然保护区管理 提供科学有效的数据支撑。

\section{1 研究区概况与方法}

\section{1 研究区概况}

江西省庐山国家级自然保护区(图 1)北邻长江, 位于鄱阳湖西部, 处于鄱阳湖与长江交汇口 $\left(29^{\circ} 25^{\prime}\right.$ $18^{\prime \prime} \mathrm{N} \sim 29^{\circ} 39^{\prime} 57^{\prime \prime} \mathrm{N}, 115^{\circ} 52^{\prime} 38^{\prime \prime} \mathrm{E} \sim 116^{\circ} 05^{\prime} 25^{\prime \prime} \mathrm{E}$ ), 保 护区大体以庐山山麓环山公路为界, 地跨九江市的 星子县、九江县和庐山区 3 个县区, 总面积 30493 $\mathrm{hm}^{2}$ 。庐山自然保护区内植被覆盖度高达 $76 \%$, 保 护区内生物多样性丰富, 为亚热带常绿榈叶林区。 庐山具有自然与人文的双重属性, 旅游活动和其他 人类活动的影响深刻而持久。人类经济活动中不 合理的开发利用,使庐山的原始植被大多遭到人为 破坏, 庐山现有植被中大多为人工林, 主要植被类 型包括常绿阔叶林、常绿落叶阔叶混交林、落叶阔 叶树和针叶林。

\section{2 数据源及处理}

\subsection{1 植被指数数据}

本文研究所采用的数据为欧洲航天局(http:// www.vito-eodata.be/)Proba- V 卫星提供的 S5 TOC NDVI $100 \mathrm{~m}$ [C1]植被指数产品,共计 288 幅, 时间 范围为 2015-2018 年, 时间分辨率为 $5 \mathrm{~d}$, 空间分辨 率为 $100 \mathrm{~m}$ 。

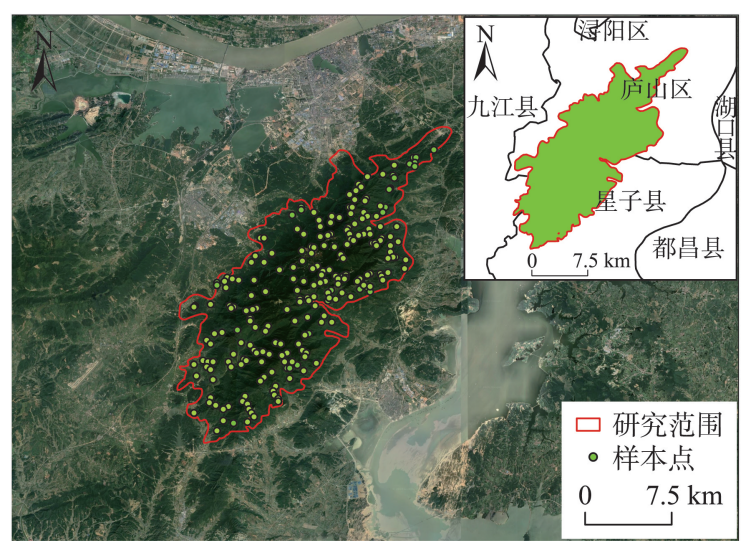

图 1 研究区位置及采样点分布

Fig.1 Location of the study area and distribution of the sampling sites 
利用研究区边界截取庐山自然保护区NDVI时 序数据集, 根据下面公式将 DN 值转化为对应的标 准NDVI真实值:

$$
\mathrm{PV}=(\mathrm{DN}-\mathrm{OFFSET}) / \mathrm{SCALE}
$$

式中:PV 为标准 NDVI值; DN 为遥感影像像元亮度 值; 根据在 Proba-V 卫星提供的 NDVI 产品文档, OFFSET 值为 20 , SCALE 值为 250 。

为尽量减少云层、大气等对 NDVI时间序列数 据的影响 ${ }^{[21]}$, 本文采用最大值合成法(Maximum Value Composite, MVC) 对庐山自然保护区的 $5 \mathrm{~d}$ 一景 的 NDVI 时序数据按月进行合成, 每年生成 12 个月 的月 NDVI产品数据。但是, 当一月中的所有数据 都受到云的干扰时, 仅使用 MVC 最大值合成法进 行去云处理仍无法得到理想的效果 ${ }^{[22]}$ 。因此, 还需 要对时序数据做进一步的处理。为进一步降低噪 声和云的干扰以达到提高数据质量的目的, 本文采 用时间序列谐波分析方法(HANTS) 对研究区的 NDVI 时序数据进行重建。由于有些年份的月最大 值合成植被指数数据存在异常值或者无效值, 因此 使用相邻年份的月最大值合成数据进行补充, 从而 减少由数据缺失对最终结果的影响。HANTS 是基 于傅里叶变换改进的一种时序数据处理的有效算 法, 它将时间序列分解为平均值和多个不同频率的 正弦函数(谐波)。使用 ENVI遥感图像处理软件中 的 HANTS 工具对每一年合成的多波段影像文件进 行平滑降噪, 其主要参数设置包括 DOD(Degree of Determinedness)、拟合误差FET(Fit Error Tolerance)、 频率个数NOF(Number of Frequencies)等。

\subsection{2 样点数据}

本文对庐山保护区矢量边界范围进行分类研 究, 分类中所使用的训练和验证数据主要由 2 个部 分构成:一部分来自 2017 年 8 月实验组实地调查获 得的地面林地类型数据(约 50 个采样数据), 采用定 位拍照的方式记录地面林地类型, 并估算覆盖度; 另一部分则来自于 2017 年庐山自然保护区的森林 林斑调查, 约有 3300 条调查记录, 其中数据内容主 要包括林地面积、林地类型、森林覆盖率等。

\section{3 研究方法}

\subsection{1 分类方法}

随机森林算法是由美国科学院院士 Leo Breiman 在 2001 年提出 ${ }^{[23]}$ 。随机森林算法采用自助法 重采样技术, 从原始训练样本集 $\boldsymbol{M}$ 中有放回地重复 随机选取 $N$ 个样本生成新的训练数据集, 然后根据
自助采样获取的样本集生成由 $N$ 个决策树组成的 随机森林, 最后由 $N$ 棵决策树的预测结果, 通过投 票的方式来决定新样本的类别归属。随机森林中 的每一颗决策树都为二叉树, 其生长遵循自顶向下 的递归分裂原则, 即从根节点开始依次对训练集进 行划分。在二叉树中, 根节点包含全部训练数据, 按照节点不纯度最小原则, 分裂为左节点和右节 点, 它们分别包含训练数据的一个子集, 按照同样 的规则, 节点继续分裂, 直到满足分支停止规则, 停 止生长。该算法的基本原理如图 2 所示。

本文的随机森林算法是通过调用 $\mathrm{R}$ 软件平台 的“Random Forest”程序包实现的。使用 $\mathrm{R}$ 软件平 台从 800 组数据中随机抽取 400 组作为训练数据用 于模型的训练, 其余 400 组数据用于模型精度的评 估。随机森林算法的实现过程具有双重随机性: (1 样本选择的随机性。通过自助法重采样技术, 从 800 组原始数据集中(地面林地类型调查数据和林 斑数据) 中有放回地随机重复抽取 400 个作为训练 样本, 没有被抽中的数据称为袋外数据 $(\mathrm{OOB})$ 。(2) 特征选择的随机性。影像中的每一个波段都是一 个特征变量, 从所有的特征变量中随机选取若干个 变量建立分类决策树, 然后重复 $N$ 次上述过程, 最 后得到由 $N$ 棵决策树组成的随机森林。一般来说, $N$ 值越大, 预测效果越好, 但计算所需要的时间也越 长, 为节约计算所耗费的时间, $N$ 值只需要使 $\mathrm{OOB}$ 误差保持稳定即可, 本文将 $N$ 值设置为 400 。随机 森林算法不仅可以实现遥感影像的分类,而且在特 征选择和降维等方面也发挥着重要的作用 ${ }^{[2]}$ 。通 过袋外数据拟合误差不仅可以评估分类精度,还可 以计算各特征变量的重要性(variable importance), 以更好地进行特征选择 ${ }^{[25]}$, 从而减轻圥余信息对分

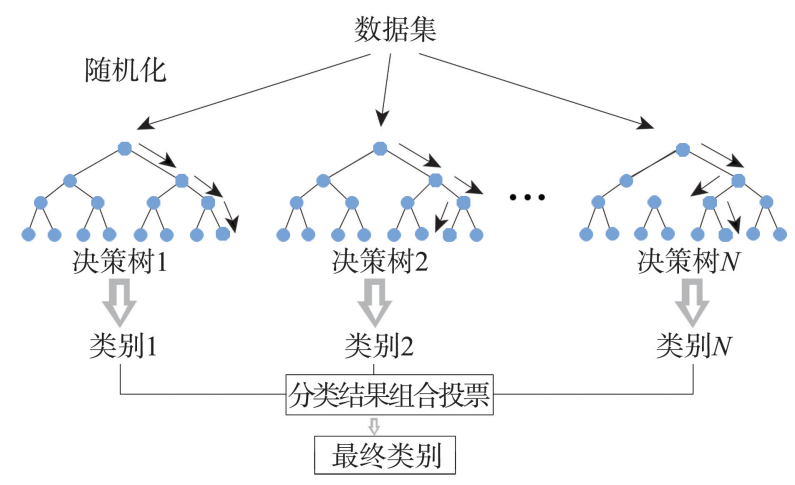

图 2 随机森林模型结构示意图

Fig.2 Schematic diagram of the random forest model 
类结果所造成的影响。本文对影像的各个特征波 段进行重要性得分排序, 选择重要性得分大于 $50 \%$ 的月 NDVI产品( $3 、 4 、 5 、 6 、 7 、 12$ 月)作为特征波段, 利用随机森林的方法进行分类。

\subsection{2 植被类别说明}

由于庐山自然保护区原始植被大多遭到人为 破坏, 现有植被大多为人工林, 且植被类型大多呈 现出聚集分布的特征。参考2017年庐山自然保护 区的林斑调查数据, 结合相关文献资料 ${ }^{[26]}$, 将庐山 自然保护区的植被类型分为阔叶林 (硬叶阔叶林和 软叶阔叶林)、针叶林(杉树林和松树林)、混交林、竹 林 4 个类别。

\section{2 结果与分析}

\section{1 庐山林地随机森林提取}

\section{1 .1 分类结果}

本文采用随机森林分类算法对庐山自然保护 区针叶林、阔叶林、混交林、竹林 4 种植被类型 2015-2018 年的 NDVI影像进行分类, 从而得到庐 山自然保护区林地分类结果(图 3)。

由图 3 可知, 2015-2018 年庐山林地植被类型 的空间分布情况。总体上看, 针叶林、阔叶林空间 分布相对较为集中, 混交林与竹林散布在他们中 间。这与 2017 年林斑数据中各林种的总体趋势一
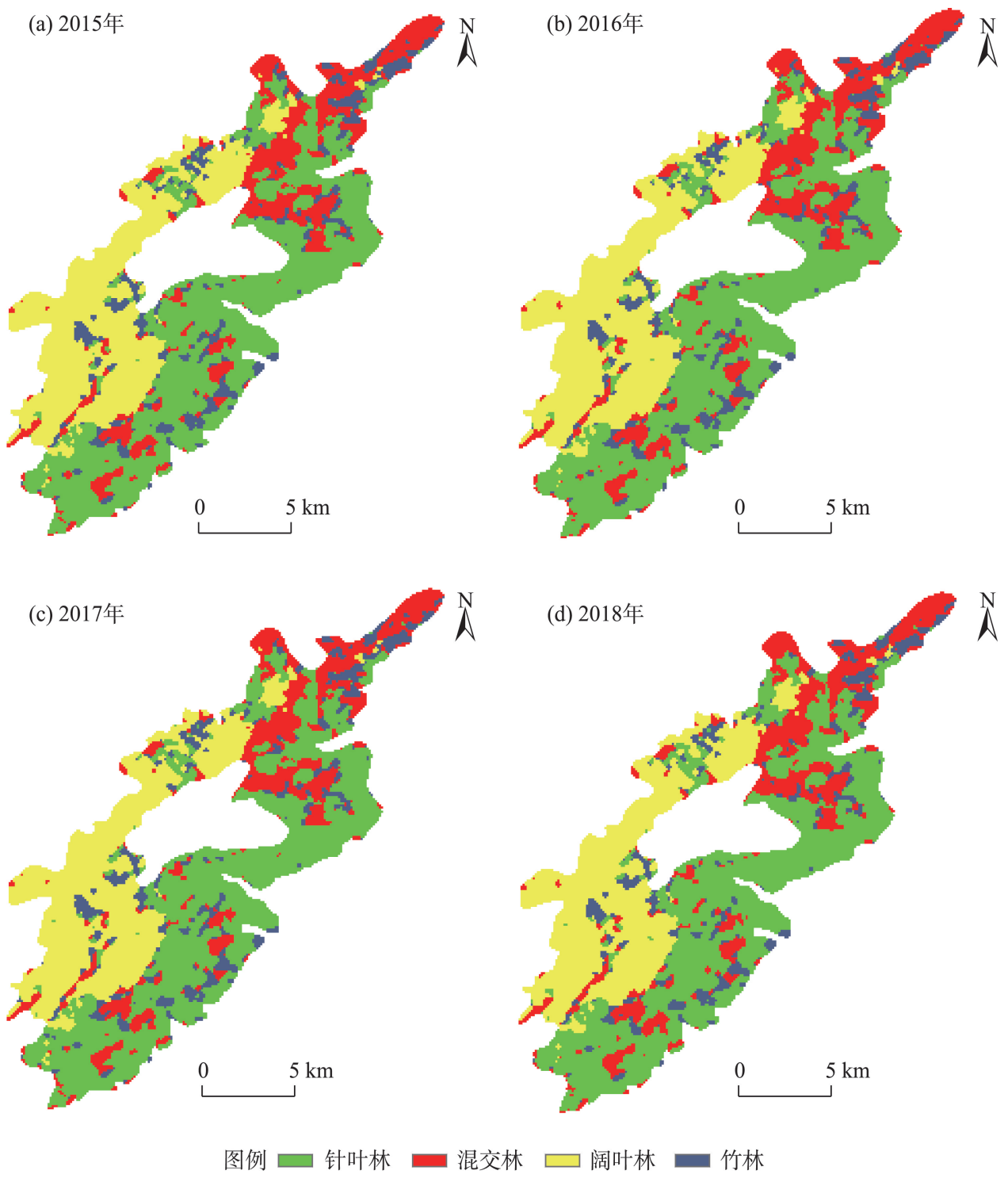

图 3 2015-2018 年庐山林地随机森林分类结果

Fig.3 Random forest classification results of Lushan forestland, 2015-2018 
致。从空间分布上看, 庐山的针叶林生长区域主要 集中在庐山的东部(汉阳峰、五老峰附近); 阔叶林主 要分布在庐山西部(黄石岩、铁佛寺周围); 而混交林 主要分布在庐山北部及阔叶林和针叶林的交错地 带; 竹林混生于阔叶林和针叶林及混交林之间, 在 山间和林地中零星或成片分布。

\section{1 .2 分类精度评价}

本文利用野外实地考察资料和 2017 年庐山自 然保护区林斑调查数据, 挑选验证样点共计 400 个。为了定量描述分类结果与实际样本之间的混 淆程度以及分类精度上的差异, 主要采用总体精 度、Kappa系数、生产者精度和使用者精度 4 个指标 来对各年份的分类结果进行评价,结果如下(表 1): 2015-2018 年的随机森林算法的总体分类精度均 较高, 分类的总体精度分别为 $85.00 \% 、 84.25 \%$ 、 $84.75 \% 、 85.25 \%$, Kappa 系数均在 0.8 左右; 从生产 者精度和使用者精度来看, 各年份的生产者和使用 者精度都在 0.8 以上, 这表明随机森林分类器在庐 山林地分类中有着较高的适用性。从庐山各年份 的林地分类精度来看, 无论是单个类别还是总体分 类都取得了较好的分类效果。

\section{2 随机森林提取结果与其他植被覆盖产品的对比 分析}

本文共选取了 LC- CCI(2010)、Globcover (2009)、Globeland30(2010)、MODIS(2010)4 种全球 植被覆盖产品与 2018 年庐山林地类型随机森林提 取结果进行对比, 如图 4所示。从林地类型上来看, 本文所提取的林地类型与 4 种植被覆盖产品都以针 叶林的分布为主, 但混交林、阔叶林在空间分布上 存在较大的差异, 这说明各植被覆盖产品在分类的 精细程度存在较大差异。

为了更好地显示本文研究所生产的植被群落 制图产品的优越性及各植被覆盖产品的差异性, 本
文将从以下几个方面进行对比分析,如表2 所示。

从空间分辨率上来看, 上述 4 种植被覆盖产品 的空间分辨率分别为 $300 、 300 、 500 、 250 \mathrm{~m}$, 而本文 所提取的庐山林地分类结果的空间分辨率皆为 $100 \mathrm{~m}$ 。本文的植被制图产品具有更高的空间分辨 率, 可以为研究庐山自然保护区植被群落的演替过 程和自然保护区管理提供更为详细的数据。

从制图产品的可获取时间上来看, 其他植被覆 盖产品在一定程度上不能完全满足保护区对一套 及时、有效的植被制图产品的需求, 本文所制备的 植被制图产品能够为掌握庐山自然保护区的植被 群落分布提供及时有效的数据。

从植被制图产品的精度而言, Globcover 产品 的总体分类精度为 $70.7 \%, \mathrm{LC}-\mathrm{CCI}$ 产品的总体分类 精度为 $74.4 \%$ 。而 2010 年 MODIS 植被覆盖产品的 准确性尚未得到评估,但 Friedl 等 ${ }^{[27]}$ 基于使用训练 数据集的交叉验证得出了其总体准确性为 $74.8 \%$ 。 Globeland 30 产品的总体分类精度达到了 $83.5 \%$ 。 而本文使用随机森林算法所提取的 2015-2018 年 庐山自然保护区的植被覆盖产品的总体分类精度 均在 $84 \%$ 以上。相对于其他植被覆盖产品而言, 本 文的制图产品具有更高的精度。

根据实地调查数据、林斑数据以及禁忠良 ${ }^{[28]}$ 的 研究结果, 竹林在庐山分布较广, 而 Globeland 30 及 其他植被覆盖产品在类别上没有将竹林这一类别 纳人分类体系中, 可见, 本文在对庐山林地类型的 划分上相对于其他植被覆盖产品而言更为精细, 在 类型划分上更为全面, 可以为掌握和管理保护区内 植被类型提供更为详实的数据资料。

\section{3 庐山林地类型面积及变化分析}

\subsection{1 林地类型面积统计}

基于 2015-2018 年庐山林地随机森林分类结 果分别提取各年份植被类型面积, 从而制作庐山林

表 1 各年份分类结果精度评价

Tab.1 Accuracy evaluation of classification results for each year

\begin{tabular}{|c|c|c|c|c|c|c|c|c|}
\hline \multirow{2}{*}{ 类别 } & \multicolumn{2}{|c|}{ 2015年 } & \multicolumn{2}{|c|}{ 2016年 } & \multicolumn{2}{|c|}{ 2017年 } & \multicolumn{2}{|c|}{ 2018年 } \\
\hline & PA & UA & $\mathrm{PA}$ & UA & $\mathrm{PA}$ & UA & $\mathrm{PA}$ & UA \\
\hline 针叶林 & 0.86 & 0.88 & 0.84 & 0.86 & 0.84 & 0.86 & 0.83 & 0.89 \\
\hline 混交林 & 0.85 & 0.83 & 0.83 & 0.84 & 0.82 & 0.84 & 0.87 & 0.84 \\
\hline 阔叶林 & 0.83 & 0.86 & 0.83 & 0.83 & 0.82 & 0.85 & 0.89 & 0.83 \\
\hline 竹林 & 0.86 & 0.83 & 0.87 & 0.84 & 0.91 & 0.84 & 0.83 & 0.85 \\
\hline $\mathrm{OA} / \%$ & \multicolumn{2}{|c|}{85.00} & \multicolumn{2}{|c|}{84.25} & \multicolumn{2}{|c|}{84.75} & \multicolumn{2}{|c|}{85.25} \\
\hline Kappa系数 & \multicolumn{2}{|c|}{0.80} & \multicolumn{2}{|c|}{0.79} & \multicolumn{2}{|c|}{0.81} & \multicolumn{2}{|c|}{0.80} \\
\hline
\end{tabular}

注: 表中PA、UA、OA 分别代表生产者精度、使用者精度、总体分类精度。 

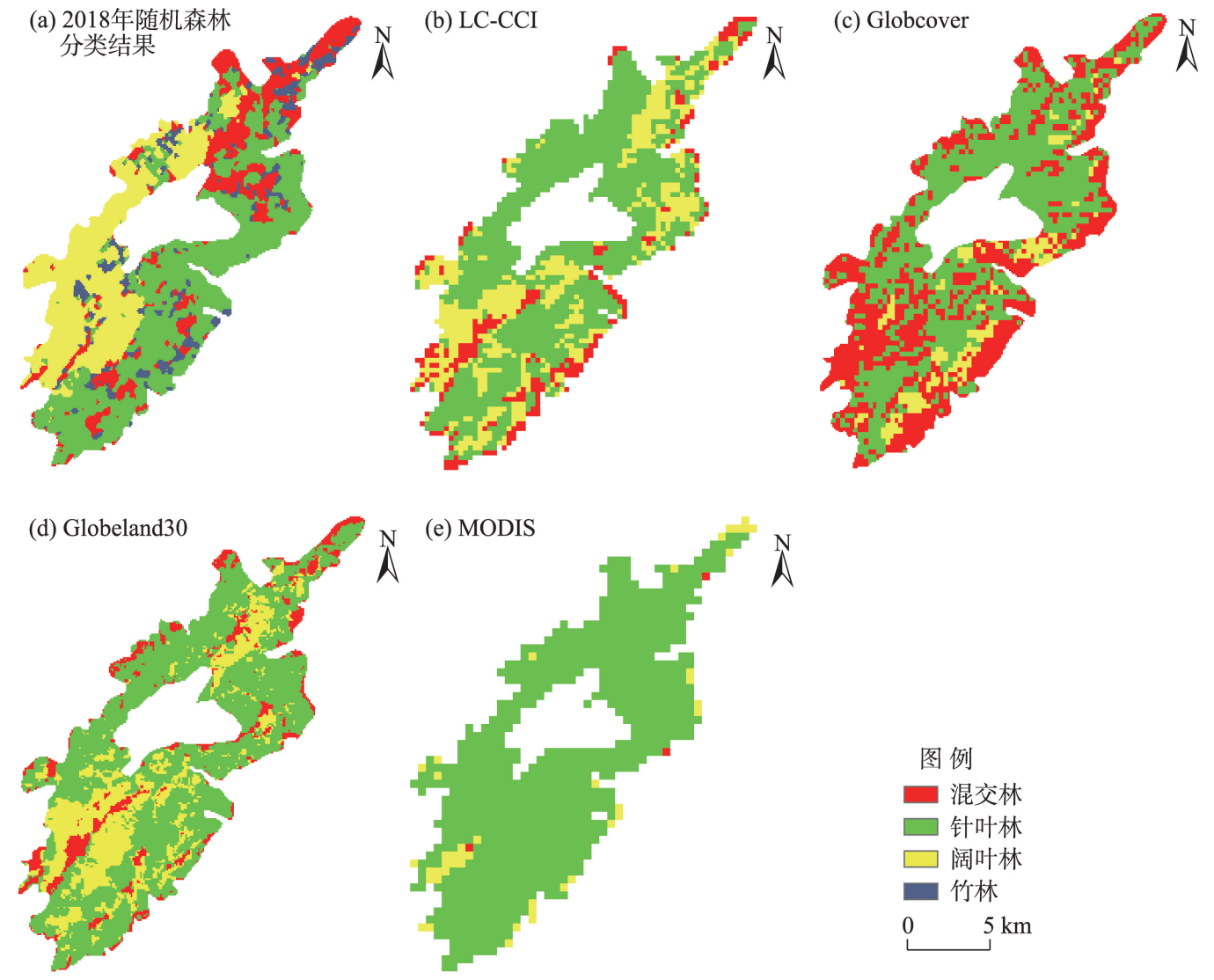

图 4 庐山林地分类结果与其他植被覆盖产品对比

Fig.4 Comparison of forestland classification results and other vegetation cover products of Lushan

表 2 本文植被制图产品与其他植被覆盖产品的概要

Tab.2 Summary of vegetation mapping products and other vegetation cover products

\begin{tabular}{|c|c|c|c|c|c|}
\hline 土地覆盖产品 & Proba-V & LC-CCI & Globcover & Globeland30 & MODIS \\
\hline 空间分辨率/m & 100 & 300 & 300 & 250 & 500 \\
\hline 数据源 & $\begin{array}{l}\text { Proba-V时间 } \\
\text { 序列数据 }\end{array}$ & $\begin{array}{l}\text { SPOT-VGT 时间 } \\
\text { 序列数据 }\end{array}$ & $\begin{array}{l}\text { MERIS卫星 } 10 \mathrm{~d} \\
\text { 合成产品 }\end{array}$ & $\begin{array}{l}\text { Landsat TM、ETM+和 } \\
\text { HJ-1 多光谱图像 }\end{array}$ & $\begin{array}{l}\text { MODIS 月 EVI、 } \\
\text { LST } 8 \text { d复合数据 }\end{array}$ \\
\hline 产品可获取时间 & 2015-2018年 & 2008-2012年 & 2009年 & 2009-2011年 & 2010年 \\
\hline 分类方法 & 随机森林分类 & $\begin{array}{l}\text { 非监督的时空聚类; } \\
\text { 机器学习分类 }\end{array}$ & $\begin{array}{l}\text { 非监督时空聚类; } \\
\text { 基于专家经验的标签 }\end{array}$ & $\begin{array}{l}\text { 基于像素和对象的分类; 基于 } \\
\text { 知识的交互验证的集成 }\end{array}$ & 决策树 \\
\hline 总体分类精度/\% & $>84$ & 74.4 & 70.7 & 83.5 & 74.8 \\
\hline
\end{tabular}

地类型面积折线图(图 5)。2015-2018 间,庐山自 然保护区内针叶林、阔叶林、混交林、竹林平均分布 面积分别为 $87.86 、 57.14 、 35.14 、 18.28 \mathrm{~km}^{2}$ 。由图 3 和图 5 可知,在所有年份中, 针叶林是庐山自然保护 区内分布最广的林地类型,竹林的分布面积最少。 总的来看, 针叶林、混交林的分布面积在 20152018 年间波动增加, 阔叶林的分布面积保持平稳, 竹林的分布面积在这期间则波动下降。

\subsection{2 林地类型变化分析}

目前国内外针对土地利用变化的研究主要是
在地理信息技术的支持下,通过对不同时期的遥感 影像数据或分类制图数据进行叠加计算从而得到 土地变化转移矩阵 ${ }^{[29]}$ 。为了更好地了解和掌握庐 山林地类型内部的转化情况,本文基于 2015-2018 年的庐山林地随机森林分类结果进行转移矩阵分 析, 了解庐山内部林地类型的变化情况(表 3 )。

根据图 3 和表 3 可知, 2015-2018 年间庐山林 地类型的变化过程具有以下特点:庐山各林地类型 之间皆存在相互转化的过程, 但其转化面积大多小 于 $1 \mathrm{~km}^{2}$, 各林地类型的转化幅度较小; 从 3 个时间 


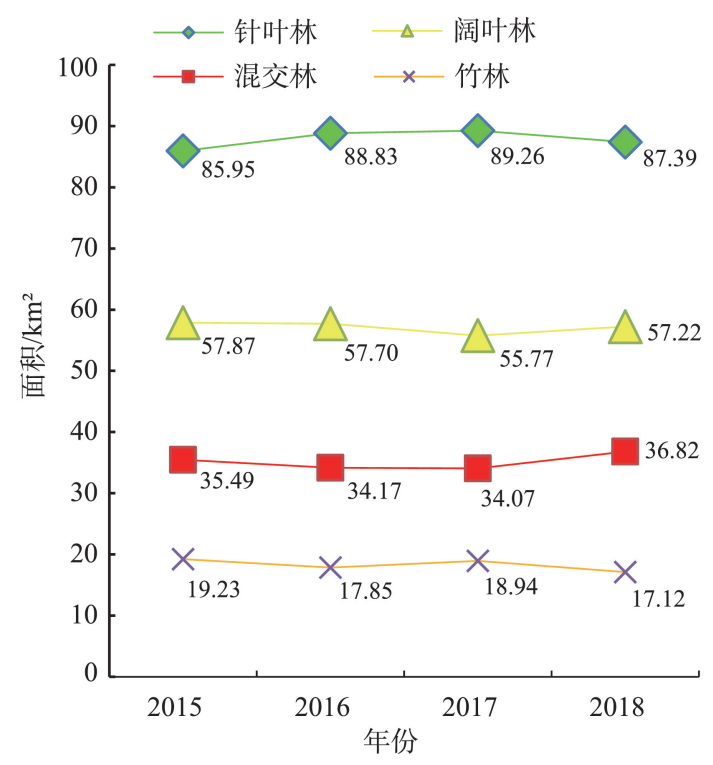

图 5 2015-2018年庐山林地类型面积折线图

Fig.5 Areas of different types of forestland of Lushan, 2015-2018

段来看, 针叶林、阔叶林、混交林的变化率皆低于 $10 \%$, 其中阔叶林变化率最低, 混交林与竹林变化 率较大;竹林的变化率相对于其他林地类型的变化 率来说, 其变化幅度较大, $4 \mathrm{a}$ 间其变化率呈现波 动, 主要与混交林相互转化。

从总体来看, 在 2015-2018 年间, 大部分庐山 各林地类型的变化率都控制在 $10 \%$ 以下, 这说明在
保护区内没有发生大范围的植被变化现象。竹林 因其属于速生型植被, 其变化率相对于其他林地类 型的变化来说变化幅度较大, 可能与其自身的生长 过程及保护区内的采伐措施相关。

\section{3 讨论}

在利用遥感数据进行大面积森林植被识别的 过程中, 长时序、高频率的遥感数据非常适合于进 行植被类型的区分与提取。由于植被具有相似的 光谱特征, 且林下结构和环境复杂, 单时相的多光 谱数据无法满足识别过程中“同谱异物”的现象, 但 植被的物候特征可以很好地解决这一问题。目前 可用的光学卫星中, 中高空间分辨率的卫星数据, 由于重返周期较长和云雨的影响, 无法达到所需的 时间分辨率要求。而能够达到 $1 \mathrm{~d}$ 重访的卫星数 据, 其空间分辨率往往较低。而 $2 \mathrm{~d}$ 的时间分辨率 和 $100 \mathrm{~m}$ 空间分辨率的 Proba- $\mathrm{V}$ 卫星是迄今为止最 为理想的数据。实验表明, $100 \mathrm{~m}$ 空间分辨率数据 可以有效地对森林的针叶林、阔叶林、混交林等进 行分类, 并且达到一定的精度, 在目前已有的土地 利用数据集中, 此数据和方法提取的专题图精度最 高,且可以有效地进行定期长时序监测。但同时由 于空间分辨率的原因, 存在较多混合像元, 导致精

表32015-2018 年间庐山林地类型面积转移矩阵

Tab.3 Transfer matrix of forestland types in Lushan, 2015-2018

\begin{tabular}{|c|c|c|c|c|c|}
\hline \multirow{2}{*}{ 2015年 } & \multicolumn{5}{|c|}{ 2016年 } \\
\hline & 混交林 & 阔叶林 & 针叶林 & 竹 林 & 变化率/\% \\
\hline 混交林 & 33.97 & 0.35 & 0.81 & 1.00 & 5.99 \\
\hline 阔叶林 & 0.11 & 56.88 & 0.38 & 0.33 & 1.41 \\
\hline 针叶林 & 0.96 & 0.54 & 84.39 & 0.91 & 2.78 \\
\hline 竹林 & 0.44 & 0.08 & 0.33 & 16.97 & 4.80 \\
\hline \multirow{2}{*}{ 2016年 } & \multicolumn{5}{|c|}{ 2017年 } \\
\hline & 混交林 & 阔叶林 & 针叶林 & 竹 林 & 变化率/\% \\
\hline 混交林 & 33.57 & 0.11 & 0.91 & 0.45 & 4.37 \\
\hline 阔叶林 & 0.32 & 56.53 & 0.44 & 0.14 & 1.59 \\
\hline 针叶林 & 0.88 & 0.48 & 84.53 & 0.37 & 2.00 \\
\hline 竹林 & 1.32 & 0.52 & 0.91 & 16.82 & 14.04 \\
\hline \multirow{2}{*}{ 2017年 } & \multicolumn{5}{|c|}{ 2018年 } \\
\hline & 混交林 & 阔叶林 & 针叶林 & 竹 林 & 变化率/\% \\
\hline 混交林 & 33.70 & 0.53 & 0.94 & 1.60 & 9.09 \\
\hline 阔叶林 & 0.04 & 56.31 & 0.20 & 0.59 & 1.48 \\
\hline 针叶林 & 0.92 & 0.49 & 84.91 & 1.00 & 2.76 \\
\hline 竹林 & 0.39 & 0.11 & 0.20 & 16.38 & 4.08 \\
\hline
\end{tabular}


度无法进一步提高。目前中国的 $4 \mathrm{~d}$ 重访、 $16 \mathrm{~m}$ 空 间分辨率的高分一号数据的 WFV 模式及 $50 \mathrm{~m}$ 空间 分辨率的高分四号同步卫星数据, 都可以尝试进行 分析, 以进一步提高森林植被类型识别精度。而针 对具体区域优势树种的识别, 由于庐山的森林物种 结构较为复杂, 则需要进一步考虑采用高光谱分辨 率的卫星数据进行研究。

从庐山植被变化的角度看, 2015-2018年植被 变化幅度不大。森林植被的更替变化需要从更长 的时间尺度进行分析。由于数据条件的限制, 本文 仅对 $4 \mathrm{a}$ 的数据进行了分析, 随着目前国内外卫星 数据各项分辨率的提高, 每年定期地对庐山森林植 被群落进行监测非常必要, 也有利于长时间的变化 监测。本文通过对庐山森林植被类型分类方法的 尝试, 能够为后期庐山森林保护区定期植被监测提 供一种有效的遥感监测方法。在本文研究的基础 上, 基于多源数据的遥感提取方法进行研究, 从而 更好地为庐山森林植被遥感制图与变化研究提供 技术支撑。

\section{4 结论}

本文基于时间序列的 Proba- $\mathrm{V}$ 数据采用随机森 林算法提取庐山植被类型, 并利用转移矩阵对庐山 自然保护区 2015-2018 年间的植被分类结果进行 叠加运算, 统计各年份植被类型面积, 从而分析保 护区内植被的变化情况, 并将分类结果与其他植被 覆盖产品进行对比分析, 结果表明:

(1) 基于 Proba-V NDVI数据产品与随机森林算 法结合, 可以对庐山自然保护区的林地类型进行分 类, 相对于其他方法与产品, 本文分类结果在空间 分辨率、制图精度上都具有较大优势, 且数据易获 取, 分类精度较高。最佳特征月份为 $3 、 4 、 5 、 6 、 7 、 12$ 月的NDVI产品数据。该方法可以为保护区每年定 期的植被类型调查与变化监测提供有效的理论与 数据支撑。

(2) 对 2015-2018 年间庐山林地类型变化分析 表明, 庐山保护区针叶林分布最广, 竹林分布最 少。这 $4 \mathrm{a}$ 中, 庐山植被保护区森林变化较少, 并未 发生大规模的植被更替现象。其中针叶林、阔叶 林、混交林的变化率较低, 转化幅度较小; 而竹林由 于受到人类活动影响较大, 故应与人类活动措施结 合进行综合分析, 分析结果可为保护区的有效管理 提供数据支撑。

\section{参考文献(References)}

[1] 尤慧. 近十年东南亚森林扰动的遥感分析 [D]. 南京: 南 京信息工程大学, 2013. [You Hui. Remote sensing analysis of forest disturbance in Southeast Asia for recent decades. Nanjing, China: Nanjing University of Information Science \& Technology, 2013. ]

[2] Myers N, Mittermeier R A, Mittermeier C G, et al. Biodiversity hotspots for conservation priorities [J]. Nature, 2000, 403: 853-858.

[3] 胡美娟, 侯国林, 周年兴, 等. 庐山森林景观空间分布格 局及多尺度特征 [J]. 生态学报, 2015, 35(16): 52945305. [Hu Meijuan, Hou Guolin, Zhou Nianxing, et al. Spatial distribution patterns and multi-scale features of the Lushan forest landscape. Acta Ecologica Sinica, 2015, 35(16): 5294-5305. ]

[4] 付伟, 徐涵秋, 王美雅, 等. 南方红壤典型水土流失区植 被分类及植被类型变化的遥感评估: 以福建省长汀县河 田地区为例 [J]. 遥感技术与应用, 2017, 32(3): 546-555. [Fu Wei, Xu Hanqiu, Wang Meiya, et al. Vegetation classification and variation assessment in a typical red soil erosion area in Southern China: Hetian, Changting County of Fujian Province. Remote Sensing Technology and Application, 2017, 32(3): 546-555. ]

[5] 刘华, 陈永富,鞠洪波, 等. 美国森林资源监测技术对我 国森林资源一体化监测体系建设的启示 [J]. 世界林业 研究, 2012, 25(6): 64- 68. [Liu Hua, Chen Yongfu, Ju Hongbo, et al. Inspiration of forest resources monitoring in USA for integrated forest resources monitoring system in China. World Forestry Research, 2012, 25(6): 64-68. ]

[6] Jia K, Wei X Q, Gu X F, et al. Land cover classification using Landsat 8 operational land imager data in Beijing, China [J]. Geocarto International, 2014, 29(8): 941-951.

[7] Poursanidis D, Chrysoulakis N, Mitraka Z. Landsat 8 vs. Landsat 5: A comparison based on urban and peri-urban land cover mapping [J]. International Journal of Applied Earth Observation and Geoinformation, 2015, 35: 259269.

[8] Calleja F, Ondiviela B, Galván C, et al. Mapping estuarine vegetation using satellite imagery: The case of the invasive species Baccharis halimifolia at a Natura 2000 site [J]. Continental Shelf Research, 2019, 174: 35-47.

[9] Petropoulos G P, Kalaitzidis C, Vadrevu K P. Support vector machines and object-based classification for obtaining land- use/cover cartography from Hyperion hyperspectral imagery [J]. Computers \& Geosciences, 2012, 41: 99-107.

[10] Barnetson J, Phinn S, Scarth P. Mapping woody vegetation cover across Australia's arid rangelands: Utilising a machine- learning classification and low-cost Remotely 
Piloted Aircraft System [J]. International Journal of Applied Earth Observation and Geoinformation, 2019, 83: 101909. doi: 10.1016/j.jag.2019.101909.

[11] Luo S Z, Wang C, Xi X H, et al. Fusion of airborne discrete-return LiDAR and hyperspectral data for land cover classification [J]. Remote Sensing, 2015, 8(1): 3. doi: 10.3390/rs8010003.

[12] Heiskanen J, Kivinen S. Assessment of multispectral, -temporal and -angular MODIS data for tree cover mapping in the tundra-taiga transition zone [J]. Remote Sensing of Environment, 2008, 112(5): 2367-2380.

[13] Zhang X, Zhang M, Zheng Y, et al. Crop mapping using PROBA-V time series data at the Yucheng and Hongxing farm in China [J]. Remote Sensing, 2016, 8(11): 915. doi: $10.3390 /$ rs 8110915 .

[14] Thanh Noi P, Kappas M. Comparison of random forest, knearest neighbor, and support vector machine classifiers for land cover classification using Sentinel-2 imagery [J]. Sensors (Basel), 2017, 18(1): 18. doi: 10.3390/s18010018.

[15] Belgiu M, Drăguţ L. Random forest in remote sensing: A review of applications and future directions [J]. ISPRS Journal of Photogrammetry and Remote Sensing, 2016, 114: $24-31$

[16] 张赫林, 彭代亮, 邓睿, 等. 基于 Landsat 时间序列数据 的祁连山区域土地利用变化 [J]. 北京工业大学学报, 2017, 43(5): 665- 676. [Zhang Helin, Peng Dailiang, Deng Rui, et al. Dynamic change of land use in Qilian Mountains based on time-series Landsat image. Journal of Beijing University of Technology, 2017, 43(5): 665676. ]

[17] 周兴文. 庐山植被研究 [J]. 沈阳教育学院学报, 1999 (2): 111-114. [Zhou Xingwen. Study on the vegetation of the Lushan in China. Journal of Shenyang College of Education, 1999(2): 111-114. ]

[18] 陈世隆, 王江林, 杨建国. 庐山的植被 [J]. 自然杂志, 1980, 2(3): 204- 208. [Chen Shilong, Wang Jianglin, Yang Jianguo. Vegetation on Lushan Mountain. Nature Magazine, 1980, 2(3): 204-208. ]

[19] 张金泉. 庐山植被的垂直分带 [J]. 华南师院学报(自然 科学版), 1982, 14(1): 1-14. [Zhang Jinquan. Division of the vertical vegetation zone in Lushan Mountain. Journal of the South China Normal University (Natural Science Edition), 1982, 14(1): 1-14. ]

[20] 孙燕. 庐山自然保护区主要道路对外来植物影响研究 [D]. 北京: 北京林业大学, 2012. [Sun Yan. Alien plant species in major roadsides and trail sides of Lushan Natural Reserve, Jiangxi Province. Beijing, China: Beijing Forestry University, 2012. ]

[21] 李杭燕, 颕耀文, 马明国. 时序 NDVI数据集重建方法评 价与实例研究 [J]. 遥感技术与应用, 2009, 24(5): 596602. [Li Hangyan, Xie Yaowen, Ma Mingguo. Reconstruction of temporal NDVI dataset: Evaluation and case study. Remote Sensing Technology and Application, 2009, 24(5): 596-602. ]

[22] 王丹, 姜小光, 唐伶俐, 等. 利用时间序列傅立叶分析重 构无云 NDVI 图像 [J]. 国土资源遥感, 2005, 17(2): 2932. [Wang Dan, Jiang Xiaoguang, Tang Lingli, et al. The application of time -series Fourier analysis to reconstructing cloud-free ndvi images. Remote Sensing for Land \& Resources, 2005, 17(2): 29-32. ]

[23] Breiman L. Random forests [J]. Machine Learning, 2001, 45(1): 5-32.

[24] Biau G E R. Analysis of a random forests model [J]. The Journal of Machine Learning Research, 2012, 13(1): 1063-1095.

[25] 张否, 宫兆宁, 王启为, 等. Sentinel-2 影像多特征优选的 黄河三角洲湿地信息提取 [J]. 遥感学报, 2019, 23(2): 313-326. [Zhang Lei, Gong Zhaoning, Wang Qiwei, et al. Wetland mapping of Yellow River Delta wetlands based on multi-feature optimization of Sentinel-2 images. Journal of Remote Sensing, 2019, 23(2): 313-326. ]

[26] 万慧霖. 庐山森林生态系统植物多样性及其分布格局 [D]. 北京: 北京林业大学, 2008. [Wan Huilin. Plant diversity and its distribution pattern in the forest ecosystem of Lushan Mountain. Beijing, China: Beijing Forestry University, 2008. ]

[27] Friedl M A, Sulla-Menashe D, Tan B, et al. MODIS collection 5 global land cover: Algorithm refinements and characterization of new datasets $[\mathrm{J}]$. Remote Sensing of Environment, 2010, 114(1): 168-182.

[28] 禁忠良. 庐山植物区系的特征和植被的分布 [J]. 黄冈 师专学报, 1994, 14(1): 52-55. [Fan Zhongliang. The features of flora and the distribution of vegetation at Lushan Mountain. Journal of Huanggang Normal University, 1994, 14(1): 52-55. ]

[29] Jenson J Q, Cowen D. Principles of change detection using digital remote sensor data $[\mathrm{M}] / /$ Star J L, Estes J E, McGwire K C. Integration of geographic information systems and remote sensing. Cambridge, UK: Cambridge University Press, 1997: 1-25. 


\title{
Forest vegetation classification and its variation in Lushan Nature Reserve using Proba-V vegetation products
}

\author{
ZHANG Li', LI Bin ${ }^{1,2}$, YANG Wenjing ${ }^{1}$, LI Qiong ${ }^{3}$ \\ (1. Key Laboratory of Poyang Lake Wetland and Watershed Research, Ministry of Education, Jiangxi Normal University, \\ Nanchang 330022, China; 2. College of Oceanography and space informatics, China University of Petroleum \\ (East China), Qingdao 266580, Shandong, China; 3. Wuhan Geomatics Institute, Wuhan 430022, China)
}

\begin{abstract}
Nature reserves are the areas with the most abundant biodiversity. Conducting a survey of current forest biodiversity in Lushan Nature Reserve and understanding the distribution and changes of vegetation in the protected area is of great significance for the protection of forest resources in Lushan. In this study, we used the Proba-V satellite 100-meter resolution 5-day vegetation index product from 2015 to 2018 using the maximum synthesis and Hants time series harmonic analysis methods to smooth the data and reduce noise, and used a random forest algorithm to classify the forest vegetation. The changes of Lushan vegetation communities were also analyzed. The research results show that: 1) The overall accuracies of the random forest classification of Lushan forestland from 2015 to 2018 were $85 \%, 84.25 \%, 84.75 \%$, and $85.25 \%$ while Kappa coefficients were $0.80,0.79,0.81$, and 0.80, respectively. Compared with the Cover-CCI-2010, Globcover-2009, Globeland30, and MODIS- 2010 land cover products, the classification results by random forest classifier based on time series NDVI products have achieved good classification effect and high classification accuracy in spatial resolution and mapping accuracy. 2) In Lushan Nature Reserve, coniferous forest and broad-leaved forest are concentrated while mixed forest and bamboo forest are scattered among them. Coniferous forest has the largest distribution area, accounting for $39.36 \%$ of the total forest area, and the bamboo forest has the smallest distribution area, accounting for $14.63 \%$. 3) From 2015 to 2018, the spatial distribution of forest vegetation communities in Lushan did not change much. The change rates of broad-leaved forest, coniferous forest, and mixed forest were all less than 10\%. Bamboo forest fluctuated during 2015-2018 because of the interference of human activities while the change of broad-leaved forest was the smallest.
\end{abstract}

Keywords: Proba-V; random forest; forest vegetation mapping and classification; spatial and temporal distribution; Lushan 